\title{
MESHLESS PETROV-GALERKIN METHOD APPLIED TO AXISYMMETRIC PROBLEMS
}

\author{
I.S. Raju* and T. Chen ${ }^{\dagger}$ \\ NASA Langley Research Center, Hampton, VA 23681-000I, U.S.A.
}

\begin{abstract}
$\underline{\text { Abstract }}$
An axisymmetric Meshless Local Petrov-Galerkin (MLPG) algorithm is presented for the potential and elasticity problems. In this algorithm the trial and test functions are chosen from different spaces. By a judicious choice of these functions, the integrals involved in the weak form can be restricted to a local neighborhood. This makes the method truly meshless. The MLPG algorithm is used to study various potential and elasticity problems for which exact solutions are available. The sensitivity and effectiveness of the MLPG algorithm to various parameters such as the weight functions, basis functions and support domain radius, etc. was studied. The MLPG algorithm yielded accurate solutions for all weight functions, basis functions and support domain radii considered for all of the problems studied.
\end{abstract}

\section{Introduction}

In the past decade, several meshless methods for the solution of partial differential equations in science and engineering appeared in the literature [1-4]. Meshless methods retain all the advantages of the finite element method without most of the disadvantages such as, element locking and discontinuous derivatives of the secondary variables across the element boundaries. Atluri and $\mathrm{Zhu}$ [4] presented a new and innovative meshless approach that uses Petrov-Galerkin weight functions instead of the traditional Galerkin weighted residual method. This meshless method is 'truly' meshless. This method, referred to as the Meshless Local Petrov-Galerkin (MLPG) method, does not need

\footnotetext{
* Head, Analytical and Computational Methods Branch, Associate Fellow AIAA

† Army Research Laboratory, MS-240, Analytical and Computational Methods Branch, NASA Langley Research Center.

Copyright $\odot 2001$ by the American Institute of Aeronautics and Astronautics, Inc. No copyright is asserted in the United States under Title 17, U.S. Code. The U.S. Government has a royalty-free license to exercise all rights under the copyright claimed herein for Governmental Purposes. All other rights are reserved by the copyright owner.
}

"mesh" or "cells" either to interpolate the solution variables or to evaluate the integrals that appear in the weak form, but rather needs only nodes that are randomly distributed in the domain.

In the present work, MLPG algorithm is formulated and implemented for axisymmetric potential and elasticity problems. Various choices of the basis functions, forms of weight functions, and sizes of support domain are considered. The approximate solutions obtained by the MLPG algorithm are compared with the exact solutions for evaluating their accuracies.

\section{Axisymmetric Potential Problems}

Consider a Poisson's equation for an axisymmetric problem bounded by a toroidal domain with its cross section defined by $\Omega$ as shown in Figure I,

$$
\frac{\partial^{2} u}{\partial r^{2}}+\frac{1}{r} \frac{\partial u}{\partial r}+\frac{\partial^{2} u}{\partial z^{2}}=g(r, z) \quad \text { in } \Omega
$$

with boundary conditions

$$
u=\bar{u} \quad \text { on } \Gamma_{\mathrm{u}} \quad \text { and } \quad q=\bar{q} \quad \text { on } \Gamma_{\mathrm{q}}
$$

where $\Gamma=\Gamma_{\mathrm{u}}+\Gamma_{\mathrm{q}}$ and $q=d u / d n$. In a steady-state heat transfer problem, that is a typical Poisson's problem, $u$ is a temperature function, $q$ is heat flux through the boundary, and $\mathrm{g}$ is function of internal heat generation.

The solution for Eq. (1) is sought in a weighted residual manner as

$$
2 \pi \int_{\Omega}\left(\nabla^{2} u-g\right) \cdot v \cdot r \cdot d r \cdot d z=0
$$

where $v$ is a weight function. The factor $2 \pi$ in Eq. (3) comes from integration over the tangential coordinate $\theta$, because of axisymmetry. 


\section{$\underline{\text { Axisymmetric Elasticity Problems }}$}

The stresses $\sigma_{i j}$ and body force $f_{i}$ for an elastic continuum need to satisfy the equations of equilibrium condition

$$
\sigma_{i j, j}+f_{i}=0
$$

where comma indicates the partial differentiation with respect to the variable that follows the comma.

The boundary conditions on $\Gamma$ bounding the domain $\Omega$ are

$$
\mathbf{u}=\overline{\mathbf{u}} \text { on } \Gamma_{\mathrm{u}} \quad \text { and } \quad \mathbf{t}=\overline{\mathbf{t}} \quad \text { on } \Gamma_{\mathrm{q}}
$$

where $\mathbf{u}$ and $\mathbf{t}$ are displacement and traction vectors, respectively.

The weighted residual statements for Eq. (4) for the axisymmetric case can be written as

$$
\begin{array}{r}
2 \pi \int_{\Omega}\left(\frac{\partial \sigma_{r}}{\partial r}+\frac{\partial \tau_{r z}}{\partial z}+\frac{1}{r}\left(\sigma_{r}-\sigma_{\theta}\right)+f_{r}\right) \cdot \\
v_{r} \cdot r \cdot d r \cdot d z=0 \\
2 \pi \int_{\Omega}\left(\frac{\partial \tau_{r z}}{\partial r}+\frac{\partial \sigma_{z}}{\partial z}+\frac{1}{r} \tau_{r z}+f_{z}\right) \cdot \\
v_{z} \cdot r \cdot d r \cdot d z=0
\end{array}
$$

where $\sigma_{r}, \sigma_{\theta}, \sigma_{z}$, and $\tau_{r z}$ are stresses in cylindrical coordinates $(r, \theta, z)$ and $\mathrm{v}_{r}$ and $\mathrm{v}_{z}$ are weight functions for $r$ and $z$ directions, respectively. In this study, the weight functions $v_{r}$ and $v_{z}$ are chosen to be identical.

Since the MLPG algorithm for the potential and the elasticity problems are very similar, the potential problem is utilized to demonstrate the formulation in the following two sections.

\section{Moving Least Square Approximation}

In the present meshless method, a trial function, $u(\mathbf{R})$, with moving least squares (MLS) scheme is used to approximate (in cylindrical coordinates $(r, z)$; $\mathbf{R}=\hat{\mathbf{i}} \cdot r+\hat{\mathbf{j}} \cdot z$, the radius vector from the origin) the distribution of the potential function $u$ over a number of randomly distributed nodes as shown in Fig. 1. The trial function, $u(\mathbf{R})$, can be expressed in a series form as [1-4]

$$
u(\mathbf{R})=\sum_{k} \varphi_{k}(\mathbf{R}) \cdot \hat{a}_{k}
$$

where $\hat{u}_{k}$ is a fictitious value of $u$ at node $k$ and $\varphi_{k}(\mathbf{R})$ are the shape functions of node $k$. (Figure 2 illustrates a least squares approximation to the $\hat{u}_{k}$ values at each of the randomly distributed nodes for a 1-D case.) The shape function, $\varphi_{k}$, can be written as

$$
\varphi_{k}=\sum_{j=1}^{m} p_{j}(\boldsymbol{R}) \cdot\left[\boldsymbol{A}(\boldsymbol{R})^{-1} \boldsymbol{B}(\boldsymbol{R})\right]_{j k}
$$

with

$$
\begin{aligned}
& \boldsymbol{A}(\boldsymbol{R})=\sum_{k=\mathbf{l}}^{N} w_{k}(\boldsymbol{R}) \cdot \boldsymbol{p}^{T}\left(\boldsymbol{R}_{k}\right) \cdot \boldsymbol{p}\left(\boldsymbol{R}_{k}\right) \\
& \mathbf{B}(\mathbf{R})=\left[w_{1}(\mathbf{R}) \cdot \mathbf{p}\left(\mathbf{R}_{1}\right), \ldots w_{N}(\mathbf{R}) \cdot \mathbf{p}\left(\mathbf{R}_{N}\right)\right]
\end{aligned}
$$

where $\mathrm{N}$ is the number of the nodes in the domain of influence (see, for example, Ref. 4 ), $w_{k}(\boldsymbol{R})$ is a weight function defined later, and $p^{\mathrm{T}}(R)$ are the basis functions and are chosen to be

$$
p^{T}(R)=\{1, \quad r, z\}
$$

for linear representation $(\mathrm{m}=3)$ and

$$
\boldsymbol{p}^{T}(\mathbf{R})=\left\{1, r, z, r^{2}, r z, z^{2}\right\}
$$

for quadratic representation $(m=6)$.

Note that in Eqs. (9) and (10), the matrices $A(R)$ and $B$ $(\boldsymbol{R})$ are of size $m \times m$ and $m \times N$, respectively, and $w_{k}$ $(\boldsymbol{R})$ is the weight function associated with the node $k$. The weight function $w_{k}(R)$ is chosen such that it is nonzero over an influence sub-domain surrounding the node $k$ and zero outside of the influence domain [1-4]. In this study, the domain of influence of node $k$ is assumed to be a circle of radius $l_{k}$. The radius $l_{k}$ must be large enough to contain at least $m$ nodes in each direction to prevent the numerical singularity of the $\boldsymbol{A}$ matrix in Eq. (9). The weight function is chosen such that it is smooth and equals unity at the center of the sub-domain, and equals zero at the boundary and outside of the sub-domain. In this paper, three spline functions with $\mathrm{C}^{1}, \mathrm{C}^{2}$ and $\mathrm{C}^{3}$ continuity are used as weight functions:

For $C^{1}$ :

$$
w_{k}(\mathbf{R})=\left\{\begin{array}{cr}
1-3 \rho_{k}{ }^{2}+2 \rho_{k}{ }^{3} & 0 \leq \rho_{k} \leq 1 \\
0 & \rho_{k}>1
\end{array},\right.
$$


for $C^{2}$

$$
w_{k}(\mathbf{R})=\left\{\begin{array}{cr}
1-10 \rho_{k}{ }^{3}+15 \rho_{k}{ }^{4}-6 \rho_{k}^{5} & 0 \leq \rho_{k} \leq 1 \\
0 & \rho_{k}>1
\end{array},\right.
$$

for $\mathrm{C}^{3}$ :

$$
w_{k}(\mathbf{R})=\left\{\begin{array}{cr}
1-35 \rho_{k}{ }^{4}+84 \rho_{k}{ }^{5}-70 \rho_{k}{ }^{6}+20 \rho_{k}{ }^{7} & 0 \leq \rho_{k} \leq 1 \\
0 & \rho_{k}>1
\end{array}\right.
$$

where $\rho_{k}=d_{k} / l_{k}$ is the normalized distance and $d_{k}=\left\|\boldsymbol{R}-\boldsymbol{R}_{k}\right\|$ is the distance between center of the support sub-domain $\boldsymbol{R}_{k}$ and the general point $\boldsymbol{R}$. The functions are shown in Figure 3a for a 1-D domain. For a 2-D domain, the weight function can be illustrated as a 'smooth tent' as shown in Fig. 3 b for a $\mathrm{Cl}$ continuity spline function.

\section{MLPG Formulation}

In the current implementation of MLPG formulation, the weighted residual form of Eq. (3) is used. The essential boundary conditions are included in the weighted residual statement using a penalty method as

$$
\begin{aligned}
2 \pi\left\{\int_{\Omega}\left(\nabla^{2} u-g\right) \cdot v \cdot r \cdot d r \cdot d z\right. \\
\left.-\alpha \cdot \int_{\Gamma_{u}}(u-\bar{u}) \cdot v \cdot r \cdot d \Gamma\right\}=0
\end{aligned}
$$

where $\alpha$ is a penalty parameter which is chosen as a large number ( $1 \times 10^{16}$ is used in this study.) Using the divergence theorem, Eq. (13) can be recast (dropping the constant $2 \pi$ ) as

$$
\begin{gathered}
-\int_{\Omega}\left[\frac{\partial u}{\partial r} \frac{\partial v}{\partial r}+\frac{\partial u}{\partial z} \frac{\partial v}{\partial z}\right] \cdot r \cdot d r \cdot d z-\int_{\Omega} g \cdot v \cdot r \cdot d r \cdot d z \\
+\int_{\Gamma}\left[\frac{\partial u}{\partial r} n_{r}+\frac{\partial u}{\partial z} n_{z}\right] \cdot r \cdot v \cdot d \Gamma \\
-\alpha \cdot \int_{\Gamma_{u}}(u-\bar{u}) \cdot v \cdot r \cdot d \Gamma=0
\end{gathered}
$$

where $n_{r}$ and $n_{z}$ are the direction cosines of the normal of the domain boundary along the $r$ - and $z$-directions, respectively.
Unlike the Galerkin method where the trial and test functions are chosen from same space, the PetrovGalerkin method uses trial and test function from different spaces. In the present work, the trial functions are chosen as in Eq. (7), and the test functions are chosen so that the evaluation of integrals involved in Eq. (14) for the $k^{\text {th }}$ node is confined to a local neighborhood $\boldsymbol{R}_{k}$. This judicious choice of the test function is what makes this method truly meshless. In the present implementation, the test function, $v\left(\mathbf{R}, \mathbf{R}_{k}\right)$, is defined in a similar manner as the weight functions in Eq. (12) by replacing the $l_{k}$ with $l_{o}$ i.e.,

$$
\begin{aligned}
v\left(\mathbf{R}, \mathbf{R}_{k}\right)=w_{k}(\mathbf{R}) & =f\left(\rho_{k}\right) & 0<\rho_{k} & \leq 1 \\
& =0 & \rho_{k} & >1,
\end{aligned},
$$

where $\rho_{k}=\left\|\mathbf{R}-\mathbf{R}_{k}\right\| / l_{o}$ is the normalized distance.

For an internal node, $l_{o}$ is selected to be equal or less than the shortest distance between the node and the domain boundary (for example, nodes $p$ and $q$ in Figure 4). For a node on the domain boundary, the subdomain is a segment of a circular region (for example, node $s$ in Figure 4).

Substitution of the chosen trial and test functions in the weak form of Eq. (14) leads to

$$
[K] \cdot\{\hat{u}\}=\{f\}
$$

where

$$
\begin{aligned}
K_{k j}= & \int_{\Omega_{s}}\left(\frac{\partial \varphi_{j}(\mathbf{R})}{\partial r} \frac{\partial v\left(\mathbf{R}, \mathbf{R}_{k}\right)}{\partial r}+\frac{\partial \varphi_{j}(\mathbf{R})}{\partial z} \frac{\partial v\left(\mathbf{R}, \mathbf{R}_{k}\right)}{\partial z}\right) \cdot r \cdot d r \cdot d z \\
& +\alpha \cdot \int_{\Gamma_{s u}} \phi_{j}(\mathbf{R}) \cdot v\left(\mathbf{R}, \mathbf{R}_{k}\right) \cdot r \cdot d \Gamma \\
& -\int_{\Gamma_{s u}}\left[\frac{\partial \phi_{j}(\mathbf{R})}{\partial r} n_{r}+\frac{\partial \phi_{j}(\mathbf{R})}{\partial z} n_{z}\right] \cdot v\left(\mathbf{R}, \mathbf{R}_{k}\right) \cdot r \cdot d \Gamma
\end{aligned}
$$

and

$$
\begin{aligned}
f_{k}= & \int_{\Gamma_{s q}} \bar{q}(\mathbf{R}) \cdot v\left(\mathbf{R}, \mathbf{R}_{k}\right) \cdot r \cdot d \Gamma \\
+\alpha & \cdot \int_{\Gamma_{s u}} \bar{u}(\mathbf{R}) \cdot v\left(\mathbf{R}, \mathbf{R}_{k}\right) \cdot r \cdot d \Gamma \\
& \quad-\int_{\mathbf{\Omega}_{s}} g(\mathbf{R}) \cdot v\left(\mathbf{R}, \mathbf{R}_{k}\right) \cdot r \cdot d r \cdot d z
\end{aligned}
$$


where $\bar{q}(\mathbf{R})$ is the prescribed value of normal flux on $\Gamma_{s q}$. In Eqs. (17) and (18), the region $\Omega_{s}$ is the support domain of $v\left(\mathbf{R}, \mathbf{R}_{k}\right)$ and is assumed to be a circle of radius $l_{a}$ (see Figures 1 and 4 ). The $\Gamma_{s u}$ and $\Gamma_{s q}$ are segments of the boundary formed by the intersection of $\Omega_{\mathrm{s}}$ and $\Gamma$ (see Figure 4 nodes s and $q$ ). The region $\Omega_{\mathrm{s}}$ can be made as small as possible by a judicious choice of the test function, $v$. Numerical integration is used to evaluate the integrals involved in Eqs. (17) and (18). A 12-point Gaussian quadrature numerical integration is used in the present study.

The axisymmetric elastic problem is formulated on similar lines and hence this formulation is not presented here.

\section{Examples}

To evaluate the current MLPG algorithm for potential and elasticity problems, several patch test problems with exact solutions are considered. The sensitivity of the MLPG solution to the basis functions defined in Eq. (11) and the three weight functions $w_{k}(\boldsymbol{R})$ selected in Eq. (12) is studied. Since all of the potential and elasticity problems in this study are linear problems, the patch test models are defined with an arbitrary constant $a$. The various normalized radiuses of domain of influence $\left(l_{k} / a\right)$ and normalized radiuses of support domains $\left(l_{0} / a\right)$ are used to study their influence on the accuracy of the results.

The results of the MLPG method are compared to exact solutions. Two error norms $\left(\left\|e_{M}\right\|_{1}\right.$ and $\left.\left\|e_{M}\right\|_{2}\right)$ are used to evaluate the effectiveness of various parameters. These norms are defined as

$$
\begin{aligned}
& \left\|e_{M}\right\|_{1}=\frac{1}{M} \sqrt{\sum_{j=1}^{M}\left(u-u_{\text {exart }}\right)^{2}} \\
& \left\|e_{M}\right\|_{2}=\frac{1}{M} \sqrt{\sum_{j=1}^{M}\left\{\left(u-u_{\text {exact }}\right) / u_{\text {exact }}\right\}^{2}}
\end{aligned}
$$

where $\mathrm{M}$ is the total number of randomly distributed internal points in the domain at which the numerical solution is evaluated and compared to the exact solution. Note that these internal points are independent points and are not associated with the nodal points used in the models. A value of $\mathrm{M}=50$ is used in this study.

\section{Potential Problems}

Three potential patch tests involving Laplace( $\left.\nabla^{2} u=0\right)$, and Poisson- $\left(\nabla^{2} u=\right.$ constant $)$ equation problems are considered.

Figure 5 shows two models of the Laplace and Poisson equation problems used for the patch tests. These examples are analyzed using the MLPG algorithm for various prescribed boundary conditions for $u$ and $q$ on the boundaries.

A triangular domain for two mixed boundary value problems for Laplace equation is studied. The exact solution for this patch test problem is $u=r^{2}-2 z^{2}+3 z$. The radius of the domain of influence $l_{k}$ is set to equal to $4 a$. Two different types of choices for $l_{a}$ are considered. In the first choice, the distance from each internal node to the closest boundary is computed. These distances are used as the values of $l_{o}$ for that node. Then the smallest value of all of the $l_{o}$ values is used for all nodes on the boundaries. With this definition, the support domain radius is different for different nodes. The MLPG algorithm with different values of $l_{o}$ for each node recovered the exact solution for the Laplace problems. In the second choice, the smallest value of all the $l_{o}$ values from the first choice is used for all the nodes. Once again, the exact solution was recovered. In these studies, the effects of the radius of the domain of influence, $l_{k}$, were also evaluated by varying the radius from $4 a$ to $10 a$. The accuracy of results are insensitive to the radius of the domain of influence.

The sensitivity of the MLPG solution is studied by further varying the support domain radius $l_{o}$. The convergence of the error norm is presented in Figure 6. All of the nodes in the model were set to have the same value of $l_{o}$. The results show that, for Laplace equation, all three weight functions gave excellent solutions for both the mixed boundary values problems and for a variety of the normalized support domain radius, $l_{\sigma} / a$.

The problem involving a Poisson's equation over an Lshaped domain is considered next. The exact solution for the patch test problem is $u=r^{2}+2 z^{2}+3 z-2$. The convergence of the solution for various values of $l_{o}$ and for different boundary conditions is presented in Figure 7. Once again, the exact solution is recovered by MLPG algorithm for all idealizations demonstrating that the present MLPG algorithm is very efficient and accurate.

Finally, a heat transfer problem involving radial heat flow in a hollow circular cylinder with prescribed 
constant temperatures $T_{1}$ and $T_{2}$ at inner and outer surfaces, respectively, is considered (see Figure 8). The exact solution of the problem is $T=T_{1}-\left(T_{1}-T_{2}\right) \cdot \frac{\ln \left(r / r_{1}\right)}{\ln \left(r_{2} / r_{1}\right)}$. The model used in the MLPG algorithm for the problem with 25 randomly distributed nodes and the boundary conditions used are shown in Figure 8.

The exact solution for the temperature is a natural logarithmic function and since the exact solution cannot be represented by polynomial, a study of use of various basis functions (polynomial function) is undertaken. Three polynomial basis functions, linear, quadratic and cubic are considered. Note that the higher order basis function requires longer computer times. Figure 9 presents the error norm for various basis functions with a $\mathrm{C}^{\prime}$ weight function. The support domain radius, $l_{0}$, is set equal to $0.35 a$ for the nodes on the boundary and equal to the distance to the closest boundary for the internal nodes. Very accurate solutions are obtained for all cases studied. Figure 9 shows that the higher order basis function yielded more accurate results than the lower order basis function.

\section{$\underline{\text { Elasticity Problems }}$}

Several elasticity problems were studied to evaluate the MLPG algorithm. The elastic material properties, Young's modulus $E=100 \mathrm{GPa}$ and Poisson's ratio $v=0.3$, were used in these problems. As in the case of the potential problems, the accuracy of the results are insensitive to the radius of the domain of influence, $l_{k}$, if the value of $l_{k}$ greater than $4 a$.

First the axisymmetric elastic MLPG method was evaluated on problems involving axial tension and radial compression of a circular cylinder with different boundary conditions. Since exact solutions for these simple patch test problems can be represented by the polynomial functions, the meshless method, as expected, recovered the solutions to machine accuracy for all weight and basis functions all of the models.

\section{Làme's Cylinder}

A hollow cylinder subjected to constant internal $\left(p_{1}\right)$ and external $\left(p_{2}\right)$ pressures (Làme's Problem) is considered (see Figure 10). For the problem analyzed, internal pressure $p_{l}$, external pressure $p_{2}$, the inner radius $r_{1}=a$ and the outer radius $r_{2}=2 a$ were used. The models used for the problem are shown in Figure 10. The exact solution of the problem is

$$
\sigma_{r}=C_{1}-C_{2} / r^{2} ; \sigma_{\theta}=C_{1}+C_{2} / r^{2} ; \sigma_{z}=2 \cdot v \cdot C_{1}
$$

$$
u_{r}=\frac{1+\nu}{E} \cdot\left[C_{1} \cdot(1-2 v) \cdot r+\frac{C_{2}}{r}\right] ; u_{\theta}=u_{z}=0 ;
$$

where

$$
C_{1}=\frac{p_{1} \cdot r_{1}^{2}-p_{2} \cdot r_{2}^{2}}{r_{2}^{2}-r_{1}^{2}} ; C_{2}=\frac{p_{1}-p_{2}}{r_{2}^{2}-r_{1}^{2}} \cdot r_{1}^{2} \cdot r_{2}^{2}
$$

Figure 11 shows the variation of the error norm for the three different weight functions with the normalized domain size using quadratic basis function. All of the nodes in the model were assumed to have the same support domain radius, $l_{o}$. The error norm reduces substantially as the domain size increases for all the three weight functions considered. Figure 11 also shows that the error norm of $C^{1}$-weight functions is lower than those with $\mathrm{C}^{2}$-and $\mathrm{C}^{3}$ - weight functions. One of the reasons why $\mathrm{C}^{1}$-weight function produces less error than the $\mathrm{C}^{3}$-weight function is possibly due to the fact that $C^{1}$ function has a larger "effective radius" than the $C^{3}$ function. The effective radius is defined to be a value of $\rho_{k}$ in Eq. (12) as the value of the corresponding weight function $w_{k}(\boldsymbol{R})$ is too small to operative in the integrals in Eq. (17). For an arbitrary small threshold value of the weight function, $w_{k}(R)=0.01$, the effective radius of $C^{1}$ function is 0.94 while the $C^{3}$ function is about 0.86 (see Figure 3(a)). This suggests that for Làme's problem the effective radius appears to be influencing the accuracy of the results.

An additional study of the domain size effect is conducted using a model with 25 randomly distributed nodes (Figure 12). Two different types of choices for $l_{o}$ are considered namely equal and unequal size domain. In the first choice "equal size domain", the $l_{o}$ is kept the same for all of the nodes and equals to the smallest distance from an internal node to the closest boundary (see Figure 12). In the second choice, "unequal size domain" is used. In this choice, for each internal node the $l_{o}$ is kept as large as possible and set to be the distance of the node to the closest boundary. For each boundary node, the $l_{o}$ is kept to the distance from that node to its adjacent boundary nodes (see Figure 12). The results in Figure 11 suggest that a more accurate solution (smaller error norm) is obtained when a larger domain size is used. Thus, the unequal size domain is expected to yield a more accurate solution. This expectation is confirmed in Figure 12 with the randomly distributed node model.

The effects of the basis function are also evaluated, since the exact solution for the displacement in Lame's problem can not be represented precisely by any of the three - linear, quadratic and cubic - polynomial basis functions used. The error norm obtained with each of the choices of the basis functions is presented in Figure 13. The cubic basis function yielded the most accurate 
results while the linear basis function produced a marginally better result than the quadratic function.

\section{Rotating Disk}

The next problem studied is that of a disk with uniform thickness rotating with a speed of $\omega \mathrm{rad} / \mathrm{sec}$. as shown in Figure 14. In this problem, all the boundaries are stress free while the disk is subjected to an inertial force per unit volume that equals to $\rho \cdot \omega^{2} \cdot r$, where $\rho$ is the density of the disk material and $\omega$ is the angular velocity. The exact solution of the problem when the Poisson's ratio is equal to zero is given by [see pages 335-337, Ref. 5]

$$
\begin{aligned}
& \sigma_{r}=\frac{3}{8} \cdot \rho \omega^{2}\left(r_{1}^{2}+r_{2}^{2}-\frac{r_{1}^{2} \cdot r_{2}^{2}}{r^{2}}-r^{2}\right) \\
& \sigma_{\theta}=\frac{3}{8} \cdot \rho \omega^{2}\left(r_{1}^{2}+r_{2}^{2}+\frac{r_{1}^{2} \cdot r_{2}^{2}}{r^{2}}-\frac{1}{3} r^{2}\right) \\
& \sigma_{2}=0 ; \quad u_{z}=0 \\
& u_{r}=\frac{3}{8} \cdot \rho \omega^{2}\left[r \cdot\left(r_{1}^{2}+r_{2}^{2}\right)+\frac{r_{1}^{2} \cdot r_{2}^{2}}{r}-\frac{1}{3} r^{3}\right]
\end{aligned}
$$

where again $E$ is Young's modulus of the material.

Three models are used to study the convergence of the solution to nodal refinement (Figure 14). In the model1 , seven nodal points are placed in the $r$ direction. In the model-2, the distance between adjacent nodes is half of the distance between adjacent nodes in model- 1 $(d=\Delta / 2$.) In the model-3, the distance between adjacent nodes is a quarter of the distance in model-1 $(\mathrm{d}=\Delta / 4)$. The number of nodes in $z$ direction is kept the same for all of three models.

Figure 15 shows the effect of the nodal arrangement with constant domain size (equals to the distance between the nodes of model-3, i.e. $\left.l_{o}=\Delta / 4\right)$. The model-3 has most nodal points and produced, as expected, the most accurate solution. However, the model- 3 requires about 4 times the CPU time of model1. The effect of the basis functions is shown in Figure 16 using model-2 with $C^{1}$ weight function. The cubic basis function yielded a more accurate result than both of the linear and the quadratic basis functions. However, the error norm of the linear basis function accuracy is about $0.1 \%$.

Figure 17 shows the effect of the domain size using model-2 and a cubic basis function. The results, once again, show that the large support domain radius yields a small error norm.

\section{Concluding Remarks}

A meshless Local Petrov-Galerkin (MLPG) algorithm is presented for analyzing potential and elasticity problems in axisymmetric domains. In this method, the trial and test functions are chosen from different spaces. By a judicious choice of these functions, the integrals involved in the weak form can be restricted to a local neighborhood. This makes the method truly meshless. The MLPG algorithm is implemented and its efficiency is studied with respect to three basis functions, three different weight functions, and different sizes of local support domain.

Potential patch test problems involving Laplace and Poisson equations are used to evaluate the efficiencies of the algorithm. To evaluate the effectiveness of the MLPG algorithm for elasticity problems, several simple patch test problems, a Làme's cylinder problem and rotating disk problem, are studied.

The $C^{1}$ weight function yielded more accurate results than $\mathrm{C}^{2}$ and $\mathrm{C}^{3}$ functions for the patch test problems studied. Since the $C^{l}$ function requires lesser computing time than $\mathrm{C}^{2}$ and $\mathrm{C}^{3}$ functions, it should be used in most problems. The larger value of the support domain radius, $l_{o}$, tended to decrease the error norm. The variation of the radius of the domain of influence, $l_{k}$, does not show a significant effect on the accuracy of the result. For most problems studied, the cubic basis function yielded most accurate results. Increasing the order of the basis function substantially increases the computing time, but reduces the error norm.

In summary, the current MLPG algorithm yielded accurate solutions for all the potential and elasticity patch tests and other problems studied. A simple $C^{1}$ weight function, with a large support domain radius and a simple linear basis function are recommended for accurate results.

\section{$\underline{\text { References }}$}

[1] Nayroles, B., Touzot, G., and Villon, P., "Generalizing the finite element method: diffuse approximation and diffuse elements," Computational Mechanics, Vol. 10. (1992) pp. 307-318.

[2] Belytschko, T., Lu, Y.Y., and Gu,L., "Element-free Galerkin methods," Int. J. Numer. Methods Eng., Vol. 37. (1994) pp. 229-256.

[3] Mukherjee,Y.X., and Mukherjee,S., "On boundary conditions in the element-free Galerkin method," 
Computational Mechanics, Vol. 19. (1997), pp. 264-270.

[4] Atluri, S.N., and Zhu,T., "A new meshless local Petrov-Galerkin (MLPG) approach in computational mechanics," Computational Mechanics, Vol. 22. (1998), pp. 117-127.
[5] Saada, A. S., Elasticity: Theory and Applications, Robert E. Krieger Publishing Co., 1983.

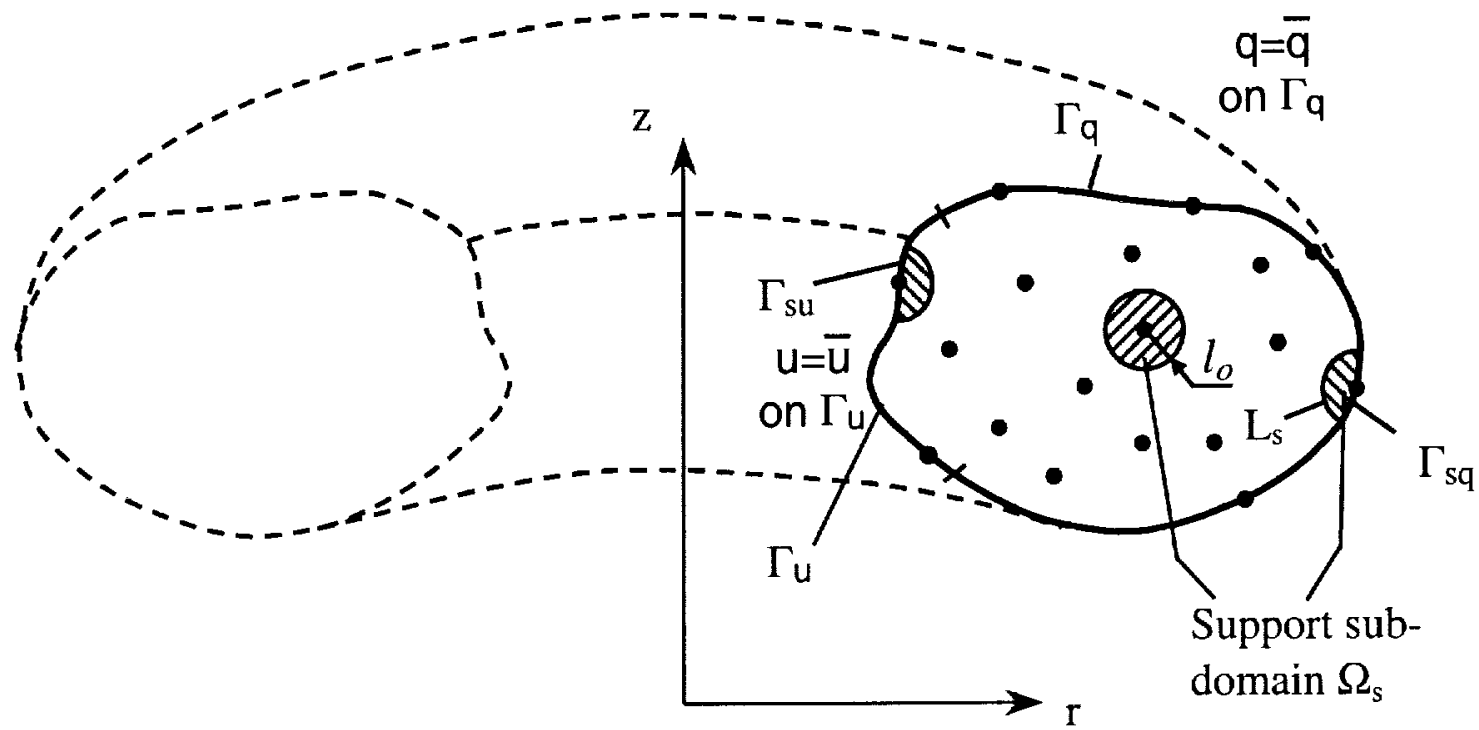

Figure 1 A typical axisymmetric potential problem

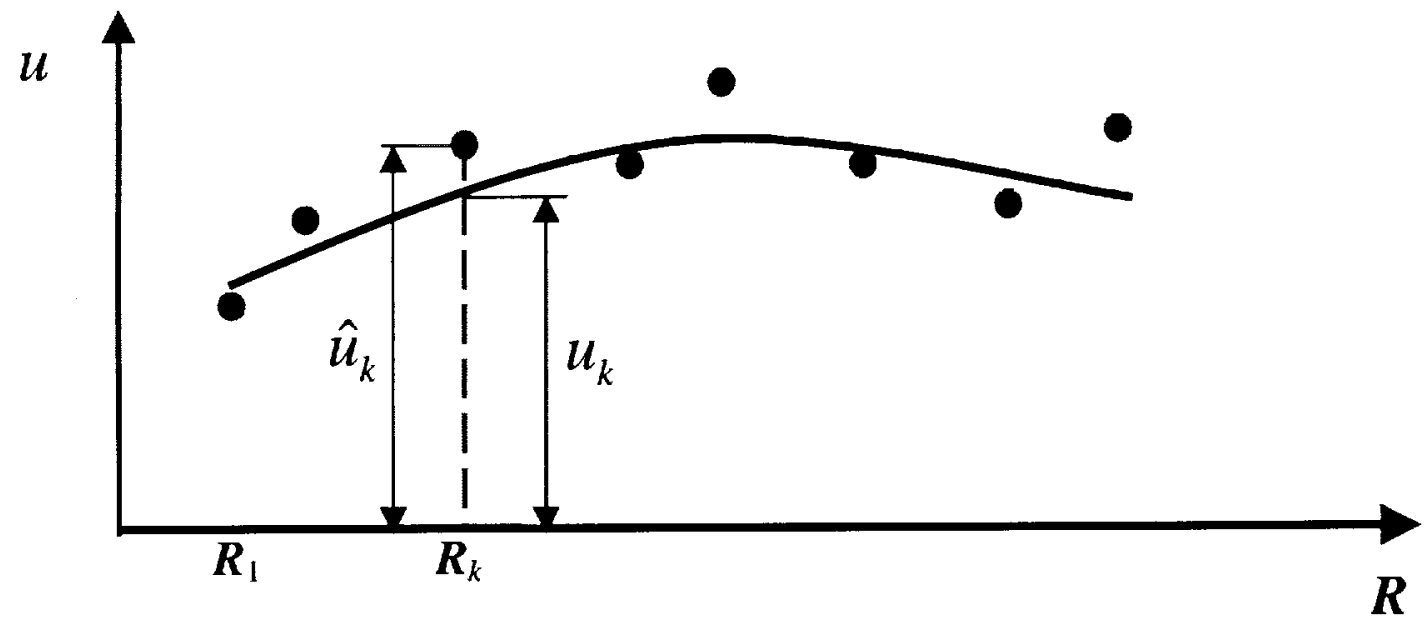

Figure 2 Approximation for $u$ using MLS scheme for a 1-D case 


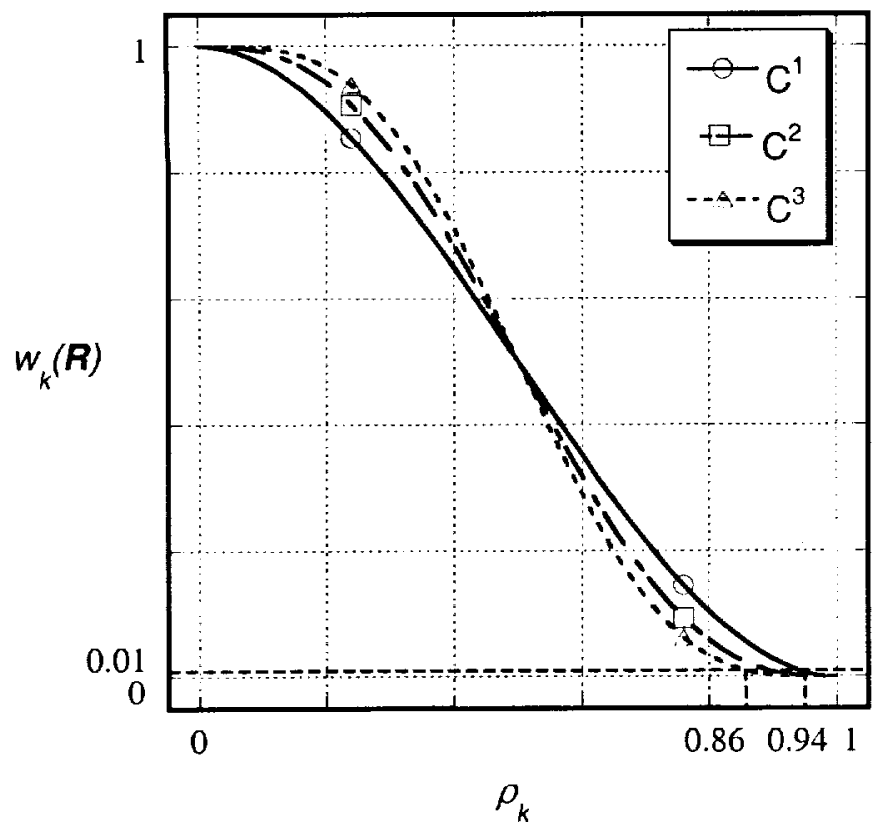

(a) Various spline weight functions used (1-D representation)

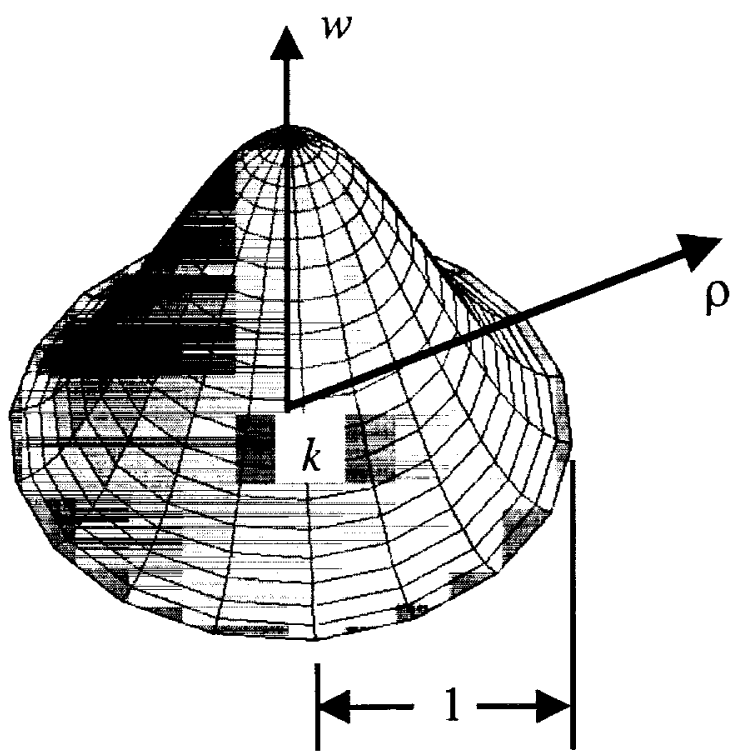

(b) $C^{\prime}$ spline weight function (2-D representation)

Figure 3 Spline weight functions used

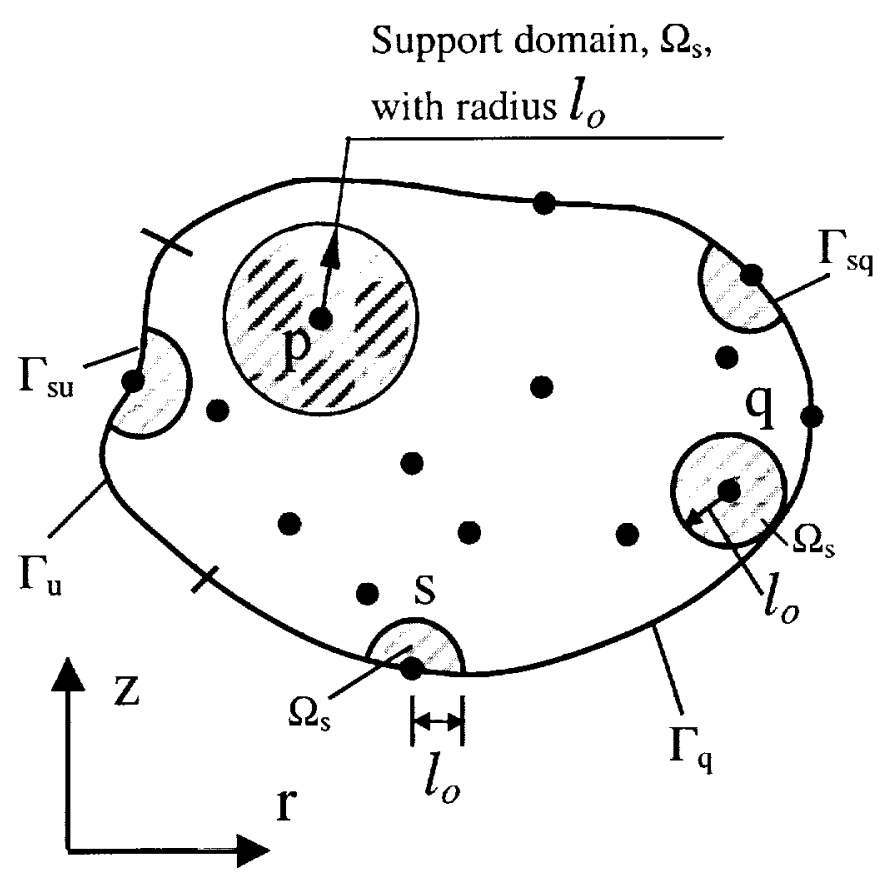

Figure 4 Convenient definitions of $l_{0}$ in the MLPG method

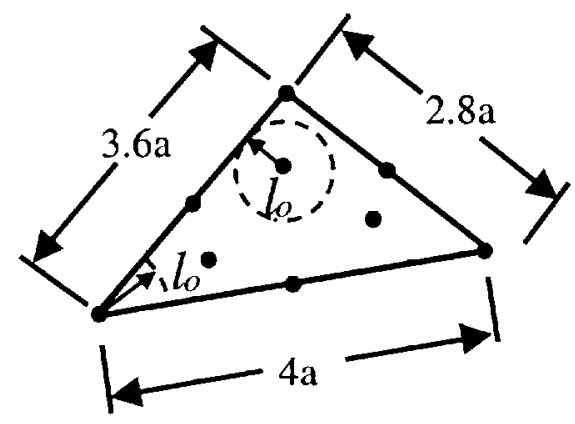

(a) Triangular domain

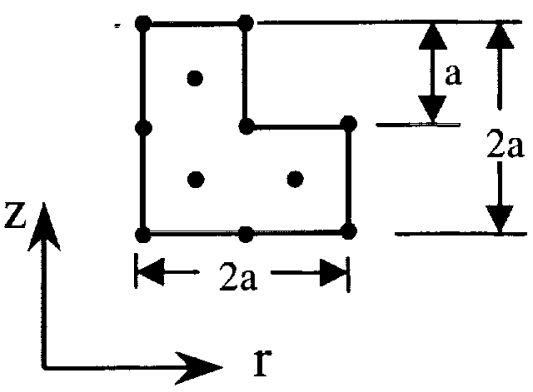

(b) L-shaped domain

Figure 5 Models used in the patch tests of Laplace and Poisson's Equations 


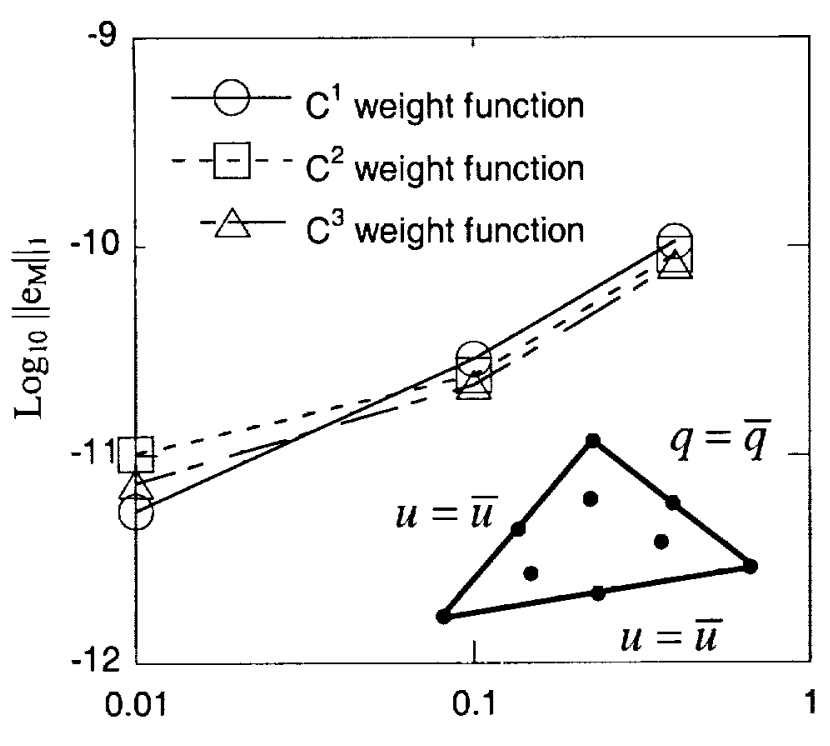

Support domain radius, $\left(l_{d} / a\right)$

(a) Mixed boundary value problem -1

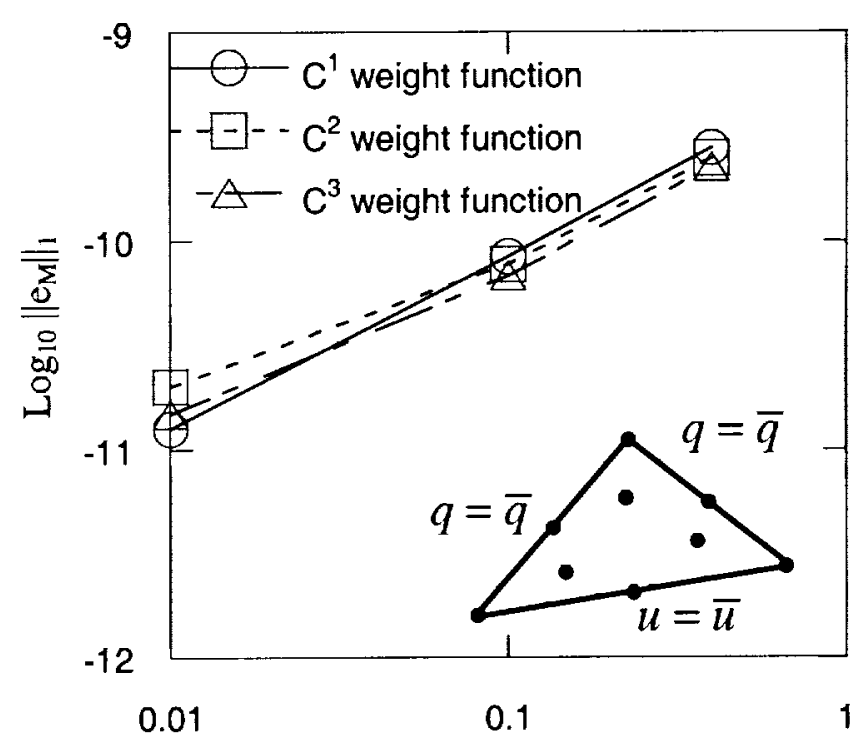

Support domain radius, $\left(l_{d} / a\right)$

Figure 6 Laplace problem involving a triangular domain - Convergence of the solution for various values of $l_{o} .\left(\nabla^{2} u=0, u_{\text {exact }}=r^{2}-2 z^{2}+3 z\right.$; Quadratic basis; $\left.l_{k}=4 a\right)$

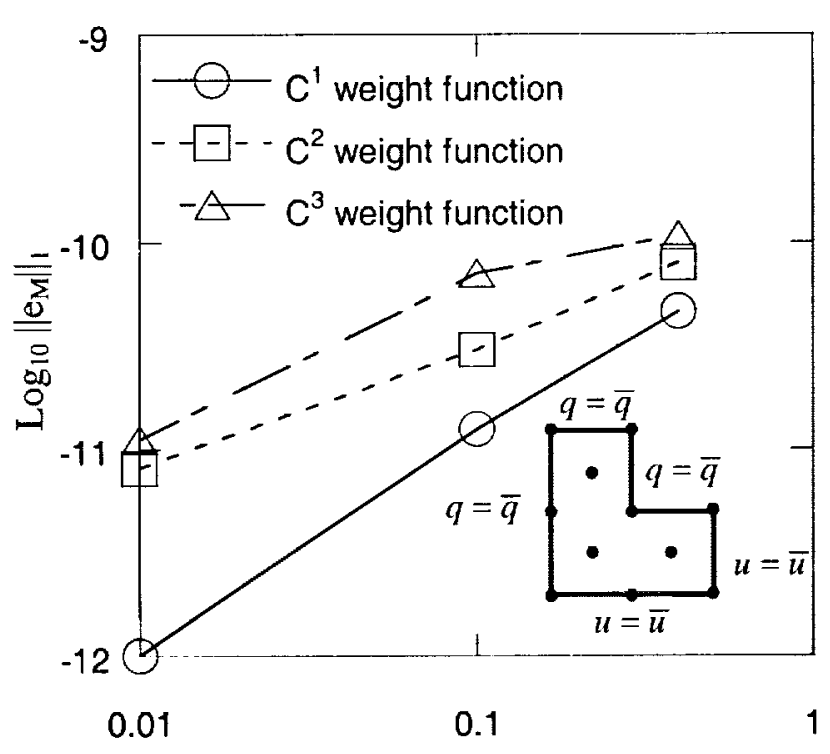

Support domain radius, $\left(l_{0} / a\right)$

(a) Mixed boundary value problem -1

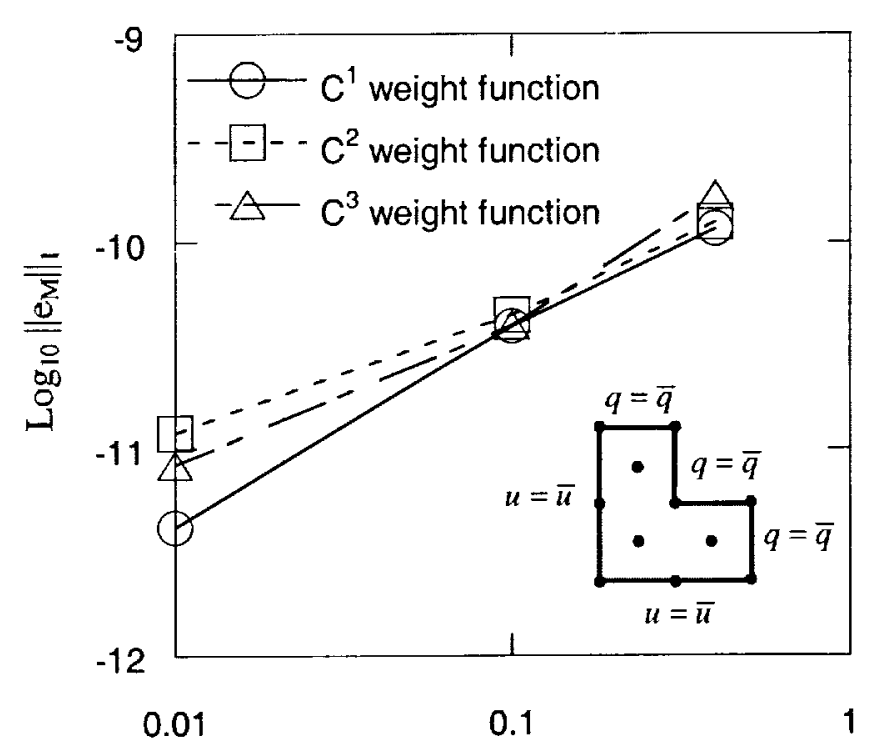

Support domain radius, $\left(l_{d} / a\right)$

(b) Mixed boundary value problem -2

Figure 7 Poisson's problem involving an L-shaped domain - Convergence of the solution for various values of $l_{o} .\left(\nabla^{2} u=8, u_{\text {exact }}=r^{2}+2 z^{2}+3 z-2\right.$; Quadratic basis; $\left.l_{k}=4 a\right)$. 


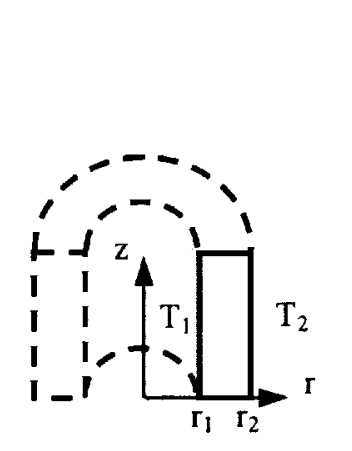

(a) Circular cylinder

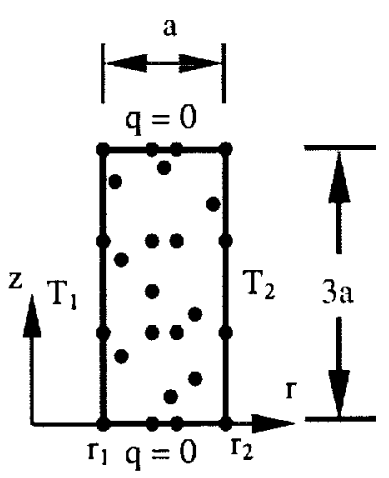

(b) Meshless model

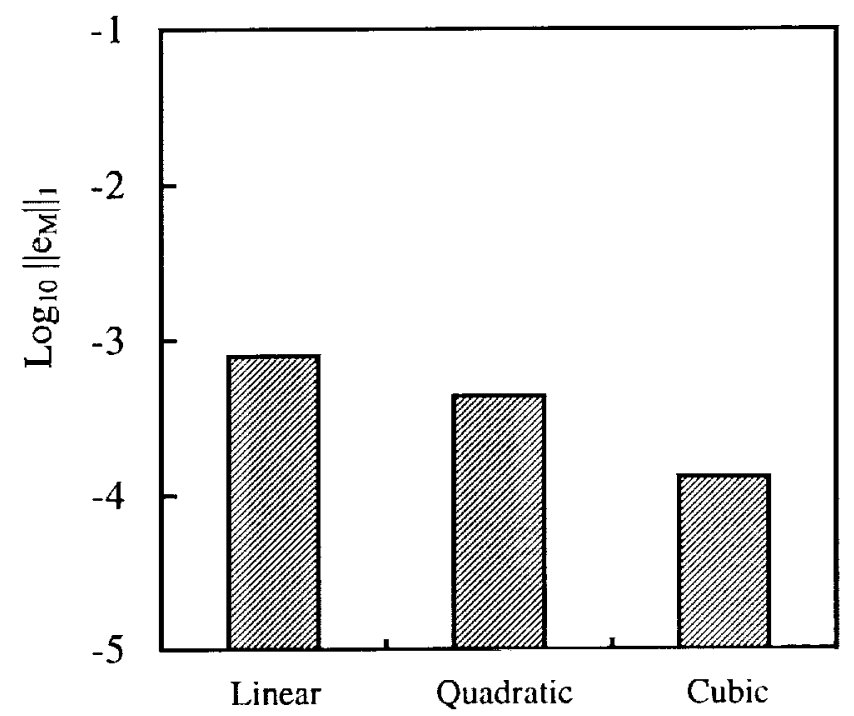

Figure 8 Heat transfer problem in a hollow circular cylinder

Figure 9 Heat transfer problem involving a hollow circular cylinder - Effect of basis functions. $\left(T_{\text {exart }}=T_{1}-\left(T_{1}-T_{2}\right) \cdot \frac{\ln \left(r / r_{1}\right)}{\ln \left(r_{2} / r_{1}\right)}, C^{1}\right.$ weight function $)$

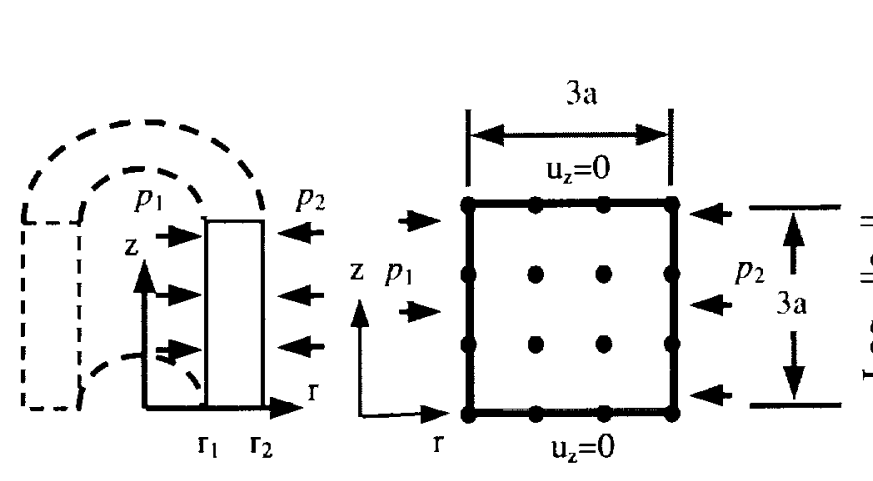

(a) Làme's cylinder

(b) Meshless model

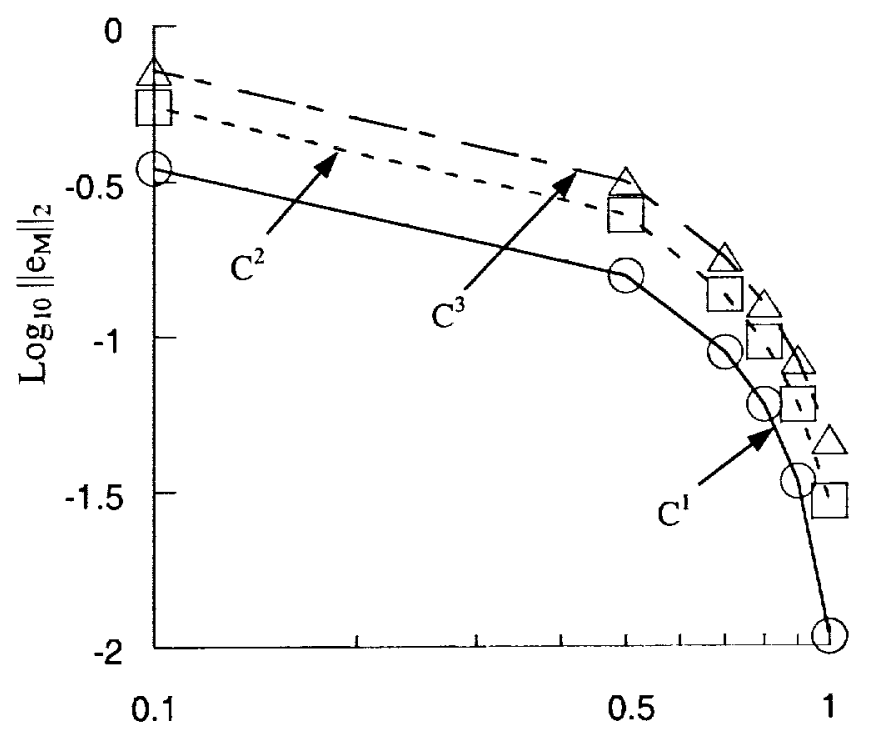

Support domain radius, $\left(l_{d} d a\right)$

Figure 10 Hollow cylinder subjected to internal and external pressures (Làme's Problem)

Figure 11 Làme's cylinder - Effects of domain radius and the weight functions 


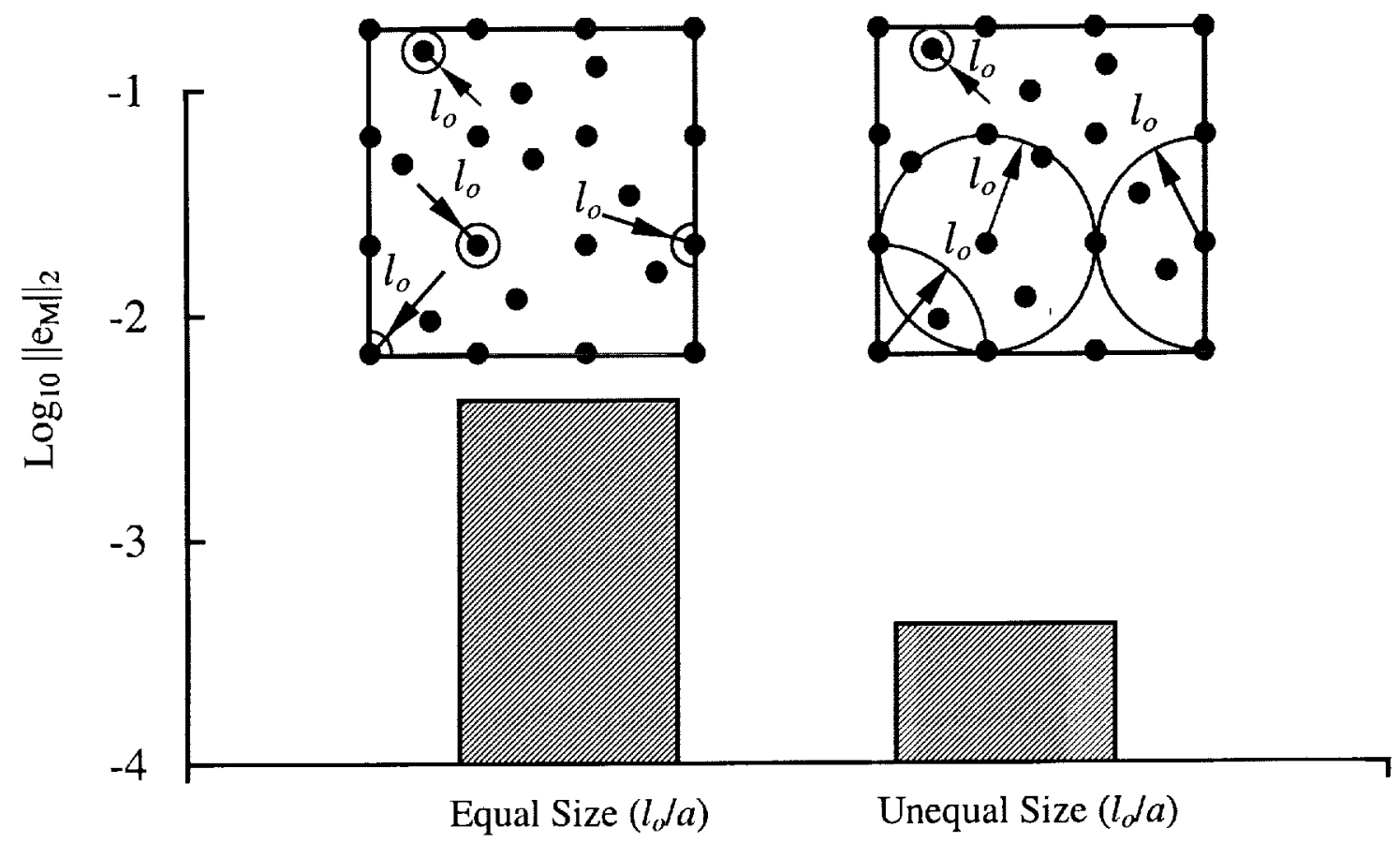

Figure 12 Làme's cylinder - Effects of support domain radius $\left(l_{0} / a\right)$ -
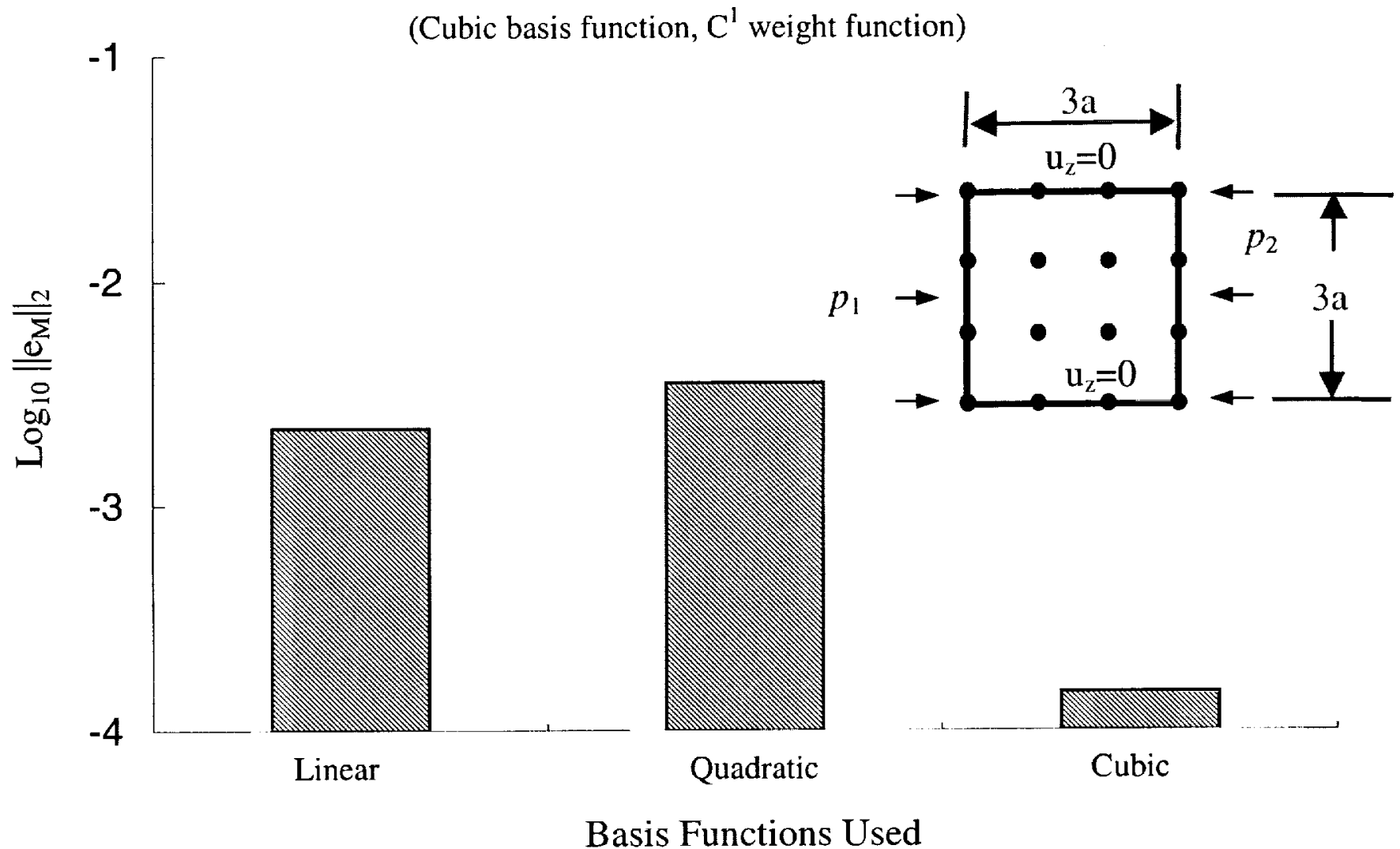

Figure 13 Làme's cylinder - Effects of basis functions $\left(C^{1}\right.$ weight function; $l_{0} / a=0.75$ ) 


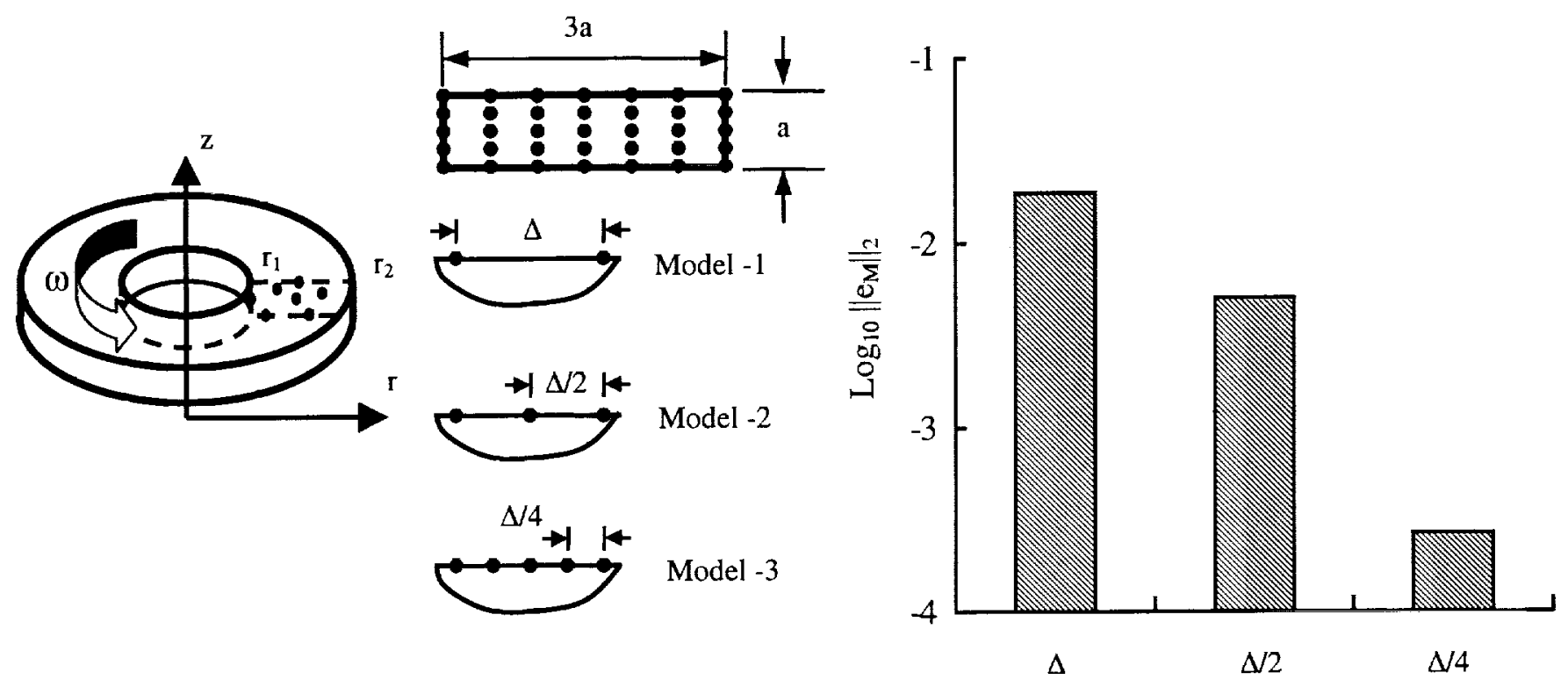

Figure 14 Rotating disk problem and the three models used

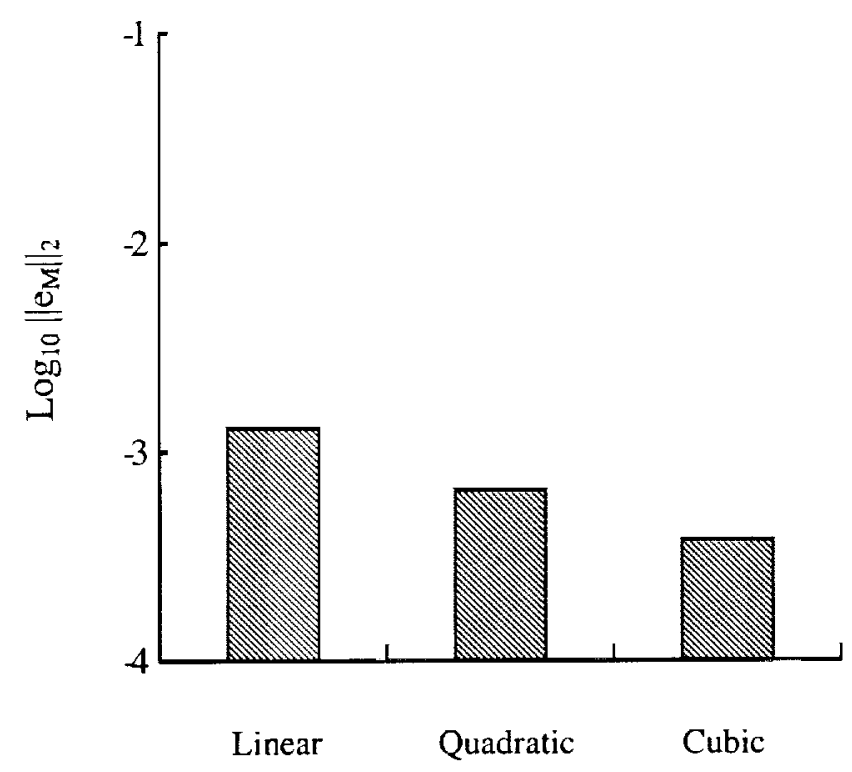

Basis functions used
Figure 15 Rotating disk - Effect of nodal refinement $\left(\mathrm{C}^{\mathrm{l}}\right.$ weight function; Cubic basis function; $l_{\alpha} / a=0.125$ )

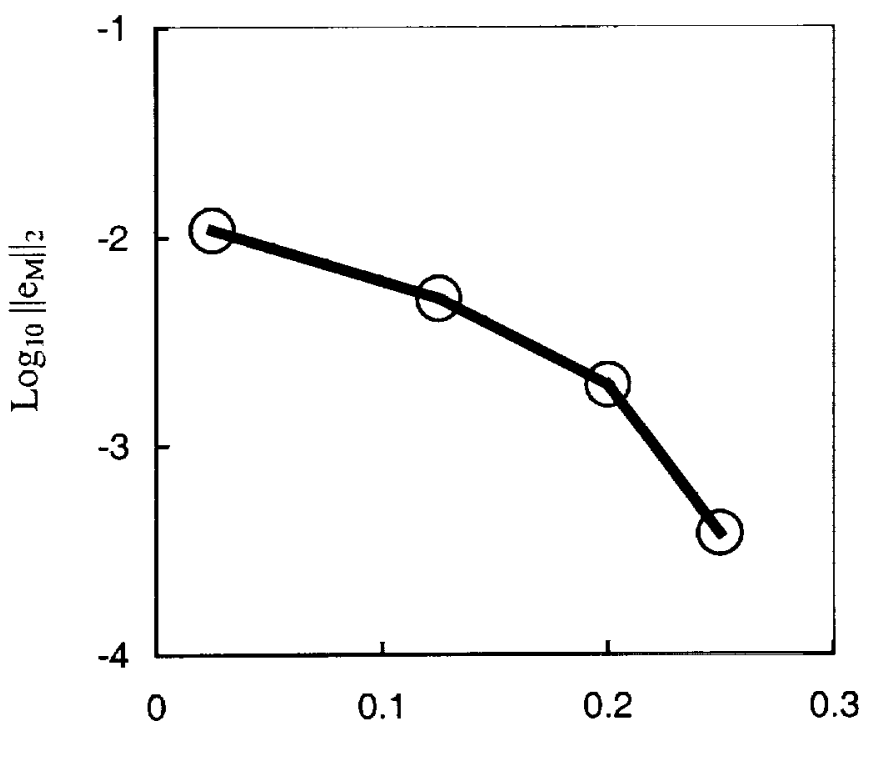

Support domain radius, $\left(l_{0} / a\right)$
Figure 16 Rotating disk - Effect of basis functions - $\left(\mathrm{C}^{1}\right.$ weight function; $l_{o} / a=0.25$; model-2)
Figure 17 Rotating disk - Effect of support domain radius - $\left(\mathrm{C}^{1}\right.$ weight function; Cubic basis function, model-2) 